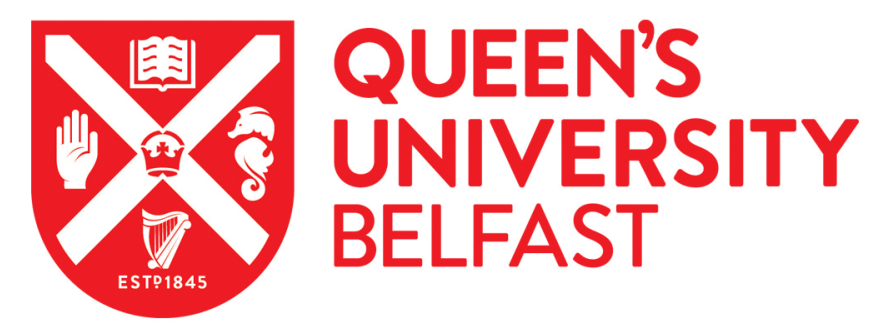

\title{
Residual stress due to curing can initiate damage in porous bone cement: experimental and theoretical evidence
}

Lennon, A., \& Prendergast, P. J. (2002). Residual stress due to curing can initiate damage in porous bone cement: experimental and theoretical evidence. Journal of Biomechanics, 35(3), 311-321.

Published in:

Journal of Biomechanics

Document Version:

Publisher's PDF, also known as Version of record

Queen's University Belfast - Research Portal:

Link to publication record in Queen's University Belfast Research Portal

\section{General rights}

Copyright for the publications made accessible via the Queen's University Belfast Research Portal is retained by the author(s) and / or other copyright owners and it is a condition of accessing these publications that users recognise and abide by the legal requirements associated with these rights.

Take down policy

The Research Portal is Queen's institutional repository that provides access to Queen's research output. Every effort has been made to ensure that content in the Research Portal does not infringe any person's rights, or applicable UK laws. If you discover content in the Research Portal that you believe breaches copyright or violates any law, please contact openaccess@qub.ac.uk. 


\title{
Residual stress due to curing can initiate damage in porous bone cement: experimental and theoretical evidence
}

\author{
A.B. Lennon, P.J. Prendergast* \\ Department of Mechanical Engineering, Trinity College, Dublin 2, Ireland
}

Accepted 17 October 2001

\begin{abstract}
Residual stress due to shrinkage of polymethylmethacrylate bone cement after polymerisation is possibly one factor capable of initiating cracks in the mantle of cemented hip replacements. No relationship between residual stress and observed cracking of cement has yet been demonstrated. To investigate if any relationship exists, a physical model has been developed which allows direct observation of damage in the cement layer on the femoral side of total hip replacement. The model contains medial and lateral cement layers between a bony surface and a metal stem; the tubular nature of the cement mantle is ignored. Five specimens were prepared and examined for cracking using manual tracing of stained cracks, observed by transmission microscopy; cracks were located and measured using image analysis. A mathematical approach for the prediction of residual stress due to shrinkage was developed which uses the thermal history of the material to predict when stress-locking occurs, and estimates subsequent thermal stress. The residual stress distribution of the cement layer in the physical model was then calculated using finite element analysis. Results show maximum tensile stresses normal to the observed crack directions, suggesting a link between residual stress and preload cracking. The residual stress predicted depends strongly on the definition of the reference temperature for stress-locking. The highest residual stresses (4-7 MPa) are predicted for shrinkage from maximum temperature; in this case, magnitudes are sufficiently high to initiate cracks when the influence of stress raisers such as pores or interdigitation at the bone/cement interface are taken into account (up to $24 \mathrm{MPa}$ when calculating stress around a pore according to the method of Harrigan and Harris (J. Biomech. 24(11) (1991) 1047-1058). We conclude that the damage accumulation failure scenario begins before weight-bearing due to cracking induced by residual stress around pores or stress raisers. (C) 2002 Elsevier Science Ltd. All rights reserved.
\end{abstract}

Keywords: Arthroplasty; Numerical model; Damage; Intramedullary prostheses; Residual stress; Cemented prostheses

\section{Introduction}

Prostheses for joint arthroplasty are often fixated into the bone using an acrylic polymer called polymethylmethacrylate (PMMA). Although it is widely used, certain aspects of the mechanical behaviour of the polymer in situ have not yet been elucidated. One such aspect is the residual stress due to shrinkage of the PMMA as it polymerises. Shrinkage stresses of sufficient magnitude could cause cracking before the joint is loaded and therefore could contribute to starting a damage accumulation failure scenario within the reconstructed joint (Huiskes, 1993). However, this has not yet been definitively demonstrated.

*Corresponding author. Tel.: + 353-1-608-1383; fax: +353-1-6795554.

E-mail address: pprender@tcd.ie (P.J. Prendergast).
Pre-load cracks have been observed in physical models (McCormack and Prendergast, 1999) and it has also been proven statistically that the rate of damage accumulation is proportional to the number of pre-load cracks (McCormack et al., 1998). The exact mechanism of stress generation during polymerisation of a two phase mixture of PMMA/MMA is difficult to ascertain. The polymerisation reaction itself is affected by several factors; first, as polymerisation progresses the mixture becomes viscous as the polymer chains grow longer and, second, the monomer remains relatively mobile leading to an auto-acceleration of the polymerisation rate (Kine and Novak, 1987). Thirdly, the reaction is highly exothermic and the release of heat further accelerates the polymerisation rate. The highly nonlinear reaction rate during the process makes it difficult to know exactly from when the material becomes capable of supporting stress. The problem is simplified greatly if the mechan- 
ism of stress-locking can be considered as a result of thermal deformations only. Some reference time from which stress-locking occurs is then needed. One such time is the peak temperature reached during polymerisation. A rationale for this is that the heat generation pulse is severe enough that thermal excitation will be sufficient to delay locking until the maximum temperature is reached.

A number of approaches have been used to estimate the level of shrinkage stress in bone cement around femoral replacements. Huiskes (1980) predicted radial temperature rise in a cement layer and calculated the residual stress due to shrinkage from the peak temperature distribution. Mann et al. (1991) assumed shrinkage from a uniformly distributed maximum temperature. Ahmed et al. (1982a) developed a model which predicted transient stresses during polymerisation, as well as locked-in stress due to shrinkage, and applied it to an axisymmetric model. However, if stress-locking occurs at the time when peak temperature is reached then stresses during the expansion phase of polymerisation, i.e. before the point of stress-locking, are likely to be relaxed and therefore not significant. If this is true, then the approach used by Huiskes (1980) is suitable for the estimation of cement residual stress. However, his calculation used a time-dependent polymerisation function which did not account for any temperature dependence in the polymerisation rate. Therefore, in his model, all regions of cement polymerised at the same rate, and this may lead to inaccuracies in the timing and magnitude of the maximum temperature. This was noted by Huiskes (1980) when comparing predictions of his model with results from an experimental study by Meyer et al. (1973).

Baliga et al. (1992) developed an empirical model for the prediction of heat generation in polymerising cement as a function of temperature and fraction of monomer polymerised. They also showed large deviations from measured behaviour if account was not taken of the dependence of polymerisation rate on local instantaneous temperature. Starke et al. (1997) implemented the model of Baliga et al. (1992) as an iterative numerical scheme suitable for finite element modelling and predicted that interior regions of cement experienced greater and more rapid temperature rise than regions nearer the interfaces - the temperature distribution was then used to predict thermal bone necrosis. Indeed, much analysis of polymerisation has been focussed on the prediction of thermal bone necrosis, (e.g. Huiskes, 1980; Starke et al., 1997), while the subject of residual stress has often been neglected because it has been assumed that residual stress will relax due to the viscoelastic properties of the cement. However, the presence of pre-load cracks in cement layers implies that the initial residual stress, although it relaxes over time, may have an immediate effect. In this paper, we use both experimental and computational models to test the hypothesis that shrinkage-induced residual stresses can cause pre-load cracking in femoral components of hip replacements. It is hypothesised that residual stresses create measurable amounts of damage. Even if residual stress later disappears due to stress relaxation, the preload damage created may initiate the damage accumulation failure scenario, as described by Huiskes and Verdonschot (1997).

\section{Methods}

\subsection{Physical model}

\subsubsection{Description and preparation}

A physical model has been developed to investigate damage accumulation around cemented femoral components of total hip replacements (Fig. 1). The model consists of a medial and lateral layer of cement encased between a layer of bone and an implant, with the whole construction held together between two aluminium covers (sideplates). The model was developed from the earlier work of McCormack and Prendergast (1999). Windows in the sideplates expose the cement layer around the stem, allowing direct observation of damage accumulation, while the sideplates themselves support the structure in a manner similar to that of cortical bone. Bovine rib bone was used to form cancellous bone margins on the inside walls of the aluminium covers. The model has proximal curvature and a trochanter-like process for the attachment of an abductor load for later studies (Lennon and Prendergast, 2001). A description of the design of the model is given in Lennon et al. (1998).

Hand mixed Simplex Rapid cement was used for all specimens as it is sufficiently translucent to allow microscopic observation of cracks by light transmission. A standard mixing ratio of $2 \mathrm{~g}: 1 \mathrm{ml}$ powder to liquid was used. Polyethylene covers were inserted into the cutouts of the inner cover to prevent cement escaping, as well as to keep the cement surface contiguous with the stem and bone surfaces. Mixing was carried out for approximately $60 \mathrm{~s}$ at $1 \mathrm{beat} / \mathrm{s}$ and the cement was introduced into the specimen cavity once a doughy state had been achieved. The specimen was then allowed to cure and was kept encased between the polyethylene covers for a further $24 \mathrm{~h}$.

\subsubsection{Crack counting}

Crack measurement was achieved by staining the sample with dye penetrant (Johnson and Allen Ltd., UK) - cracks can then be seen under magnification and light transmission through the translucent cement. Since only those cracks which intersect the exposed surfaces can be stained, this method leads to a 


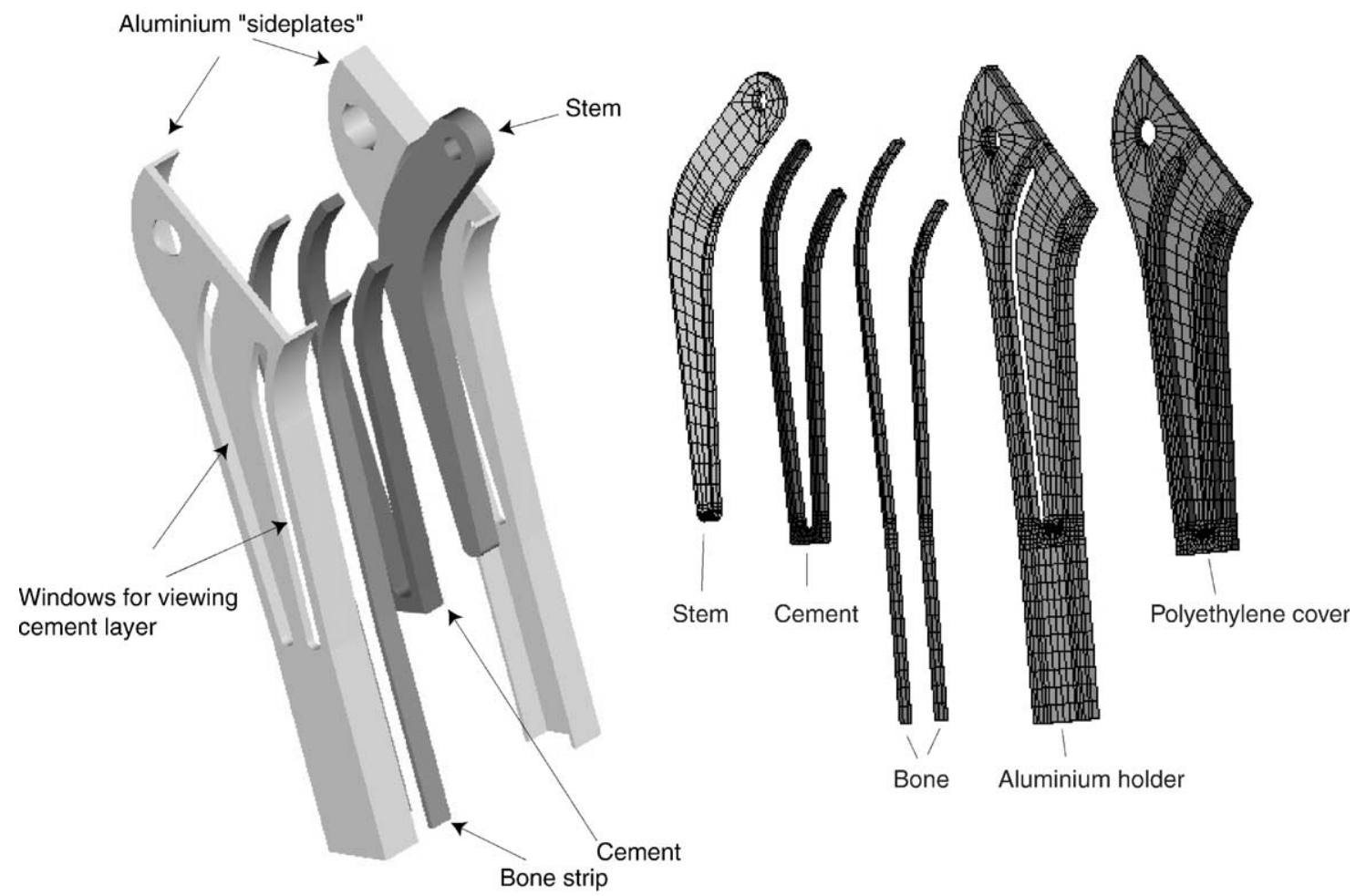

Fig. 1. Schematic view of physical model for damage accumulation study showing cut-outs for viewing of cement layers, trochanter feature with muscle attachment capability, cancellous bone strips, and aluminium side-plates. The cement thickness is $8 \mathrm{~mm}$ in the saggital plane and approximately $3-4 \mathrm{~mm}$ in the frontal plane (i.e. between implant and bone surfaces). In addition, the mesh used for the finite element study is shown on the right.

conservative count of the number of cracks as those that are entirely within the mantle cannot be stained. A Mitutoyo optical comparator with a $\times 20$ lens was used to examine the specimen for pre-cracks by imaging cracks at the specimen surface, unless it could be seen that a crack extended further below the surface - in such a case focus was changed to the plane in which the crack was clearly seen to be longest. Each crack observed was traced onto an acetate transparency from which a digital image of the crack distribution was obtained. The images were then thresholded to seperate cracks from any background level of greyscale, due to the scanning operation, and image analysis software (Image Tool, UTHSCSA, USA) was used to fit an ellipse to each crack; the major axis length, slope, and centroid were recorded for all cracks. The coordinates of the centroids were then transferred from the image coordinate system to a reference system, and the endpoints for each crack were calculated.

\subsection{Numerical model}

\subsubsection{Algorithm for heat generation and residual stress prediction}

The model presented here is an adaptation of that used by Baliga et al. (1992) and Starke et al. (1997). The polymerisation fraction $p$ of the curing mass of cement is defined as the ratio of heat generated, $Q$, at time $t$, to the total amount of heat generated on completion of polymerisation, $Q_{\text {total }}$; i.e.

$p=\frac{1}{Q_{\mathrm{total}}} \int_{0}^{t} \dot{Q} \mathrm{~d} t \simeq \frac{1}{Q_{\mathrm{total}}} \sum_{i=1}^{n} \dot{Q}_{i} \Delta t_{i}$

where the heat generation rate $\dot{Q}$ expressed per unit volume of cement, is assumed to be a function of temperature $\theta$ and polymerisation fraction $p$. The form of the heat generation rate can be approximated as

$\dot{Q}=R(\theta)\left(p-p^{2}\right)$,

where $R(\theta)$ is a rate function which depends on temperature. Baliga et al. (1992) determined, empirically, the following expression for $R(\theta)$ :

$$
\begin{aligned}
R(\theta)=4.4 \times 10^{6}\left[a_{0}\right. & +a_{1}\left(\frac{\theta}{100}\right)+a_{2}\left(\frac{\theta}{100}\right)^{2} \\
& +a_{3}\left(\frac{\theta}{100}\right)^{3}+a_{4}\left(\frac{\theta}{100}\right)^{4} \\
& \left.+a_{5}\left(\frac{\theta}{100}\right)^{5}\right]
\end{aligned}
$$

with units of $\mathrm{J} /\left(\mathrm{m}^{3} \mathrm{~s}\right)$. The coefficients $a_{i}$ are: $a_{0}=-23.89 \mathrm{~J} /\left(\mathrm{m}^{3} \mathrm{~s}\right), \quad a_{1}=296.74 \mathrm{~J} /\left({ }^{\circ} \mathrm{Cm}^{3} \mathrm{~s}\right), \quad a_{2}=$ 
$-1352.97 \mathrm{~J} /\left({ }^{\circ} \mathrm{C}^{2} \mathrm{~m}^{3} \mathrm{~s}\right), \quad a_{3}=2894.76 \mathrm{~J} /\left({ }^{\circ} \mathrm{C}^{3} \mathrm{~m}^{3} \mathrm{~s}\right), \quad a_{4}=$ $-2806.62 \mathrm{~J} /\left({ }^{\circ} \mathrm{C}^{4} \mathrm{~m}^{3} \mathrm{~s}\right)$, and $a_{5}=1009.84 \mathrm{~J} /\left({ }^{\circ} \mathrm{C}^{5} \mathrm{~m}^{3} \mathrm{~s}\right)$.

\subsubsection{Finite element model}

The mesh (Fig. 1) was generated from eight-noded hexahedral elements as a half-model, due to symmetry about the frontal plane of the specimen. ANSYS (Canonsburg, PA, USA) was used to solve both thermal and structural analyses. The polyethylene covers were included in the model (Fig. 1) for the thermal portion of the analysis but were removed for the structural analysis. This was felt to be acceptable as it was found that very little effort was required in removing the covers, implying that little or no bonding existed between the cement and polyethylene. All interfaces were assumed to be bonded for the thermal analysis and a convection load (surface convection coefficient of $2 \times 10^{-5} \mathrm{~J} / \mathrm{mm}^{2}$ ) was applied to the external surfaces of the specimen. Ambient temperature was assumed to be $23^{\circ} \mathrm{C}$ and all materials were assumed to be at this temperature at the start of the analysis. All remaining interfaces in the structural analysis were also treated as bonded. Material properties for the model are given in Table 1.

Since pores are a feature of bone cement (Murphy and Prendergast, 2000; Tepic and Soltesz, 1998), the stress distribution was also calculated using the method of Harrigan and Harris (1991). This approach assumes stress on the surface of the pore is independent of pore size and that the pore is much smaller than the region in which it is found - this leads to less than a $10 \%$ error if the distance between the pore and an interface is less than three times the pore diameter (Harrigan and Harris, 1991). The maximum stress around a pore was estimated for every element in the observable cement and averaged at each node - although it is unrealistic to assume that a pore would be present in every element, this approach allows areas of cement susceptible to crack initiation from pores to be identified.

\subsubsection{Description of the iterative procedure}

A polymerisation rate, $\Delta p_{1} / \Delta t_{1}$, was assumed for the first time increment and the required heat generation rate obtained by solving for $\dot{Q}$ in Eq. (1). For subsequent time increments, the temperature and polymerisation fraction for each cement element at the end of the increment were used to calculate the heat generation rate (from Eqs. (2) and (3)) for the next increment. Fig. 2 shows an outline of the algorithm used. The time required to achieve a $5 \%$ increase in polymerisation fraction for each element was calculated; the minimum was used as the time step so that time stepping was governed by the fastest polymerising element. Once polymerisation had completed, the time step size was increased and the analysis was stopped at $2000 \mathrm{~s}$. During the solution procedure, the centroid temperature and polymerisation fraction of each element was recorded and the maximum value was stored. The time an element finished polymerisation, as well as its temperature, was also recorded.

The next stage of the numerical modelling procedure was to determine the shrinkage stresses. These were determined for (i) shrinkage from the maximum temperature attained by each element and (ii) shrinkage from the temperature at the end of polymerisation. As the level of stress predicted will depend strongly on the amount of shrinkage that occurs, particular attention was paid to the thermal expansion coefficient. The temperature dependence of the thermal expansion coefficient of PMMA immediately after polymerisation has been approximated as a bilinear curve by Ahmed et al. (1982b) with a knee point at $43^{\circ} \mathrm{C}$ (Table 1). Therefore, using the temperature distribution obtained from the thermal analysis, the coefficient of thermal expansion was set for each element based on its temperature during each time step of the cooling phase.

\section{Results}

\subsection{Pre-load crack distributions}

Pre-load cracks were found in almost every region of the mantle over all five specimens, although there is great variability between individual specimens, see Fig. 3. Total crack length for specimens 1, 2, 4, and 5 were comparable, while specimen 3 exhibited approxi-

Table 1

Structural and thermal properties

\begin{tabular}{lllllll}
\hline Material & $\rho\left(\mathrm{kg} / \mathrm{mm}^{3}\right)$ & $\mathrm{k}(\mathrm{W} / \mathrm{mmK})$ & $c(\mathrm{~J} / \mathrm{kgK})$ & $E(\mathrm{GPa})$ & $v$ & $\alpha\left(\times 10^{-6} \mathrm{~K}^{-1}\right)$ \\
\hline Cement & $1.19 \times 10^{-6}$ & $0.18 \times 10^{-3}$ & 1450 & 2.4 & 0.33 & $72.2\left(\theta<43^{\circ} \mathrm{C}\right)$ \\
& & & & 210 & 0.33 & 12.5 \\
Stem & $7.8 \times 10^{-6}$ & $14 \times 10^{-3}$ & 460 & 2 & 0.3 & 0.1 \\
Cancellous bone & $1.3 \times 10^{-6}$ & $\left.0.29 \times 10^{-3} \mathrm{C}\right)$ \\
Aluminium & $2.8 \times 10^{-6}$ & 0.1255 & 2292 & 925 & 0.33 & 20 \\
Polyethylene & $0.92 \times 10^{-6}$ & $0.5 \times 10^{-3}$ & 1900 & 1 & 0.3 & 150 \\
\hline
\end{tabular}


THERMAL

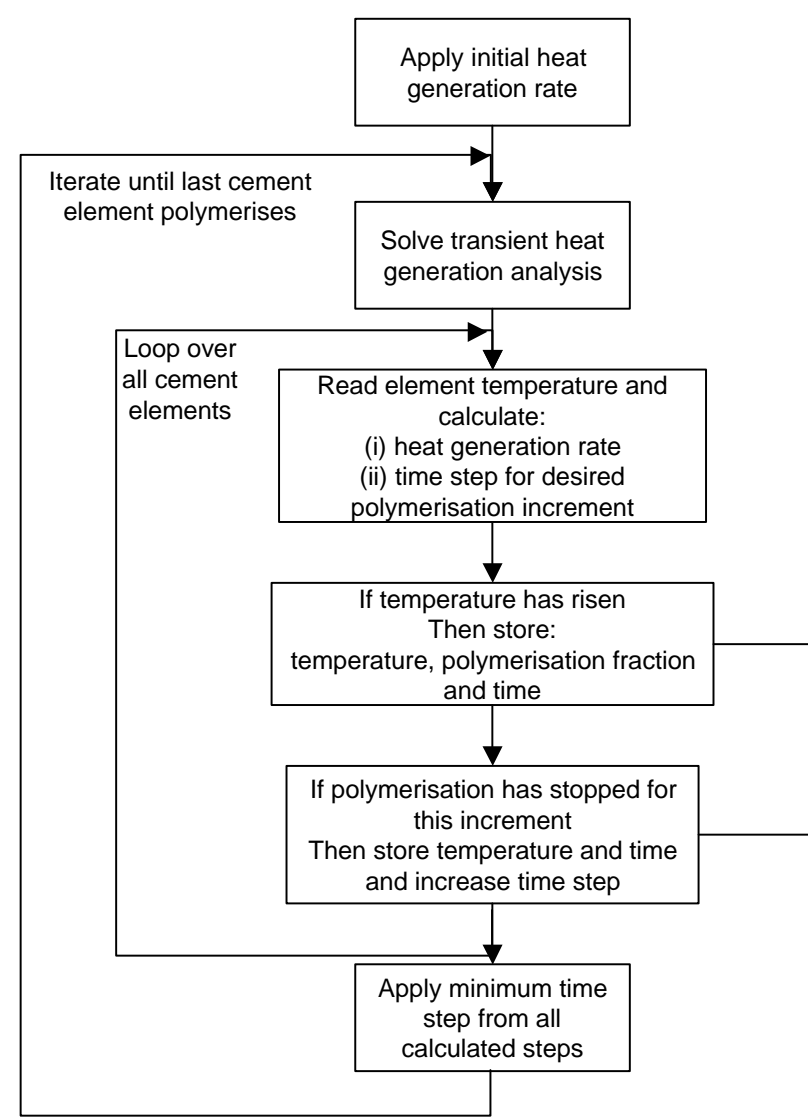

STRUCTURAL

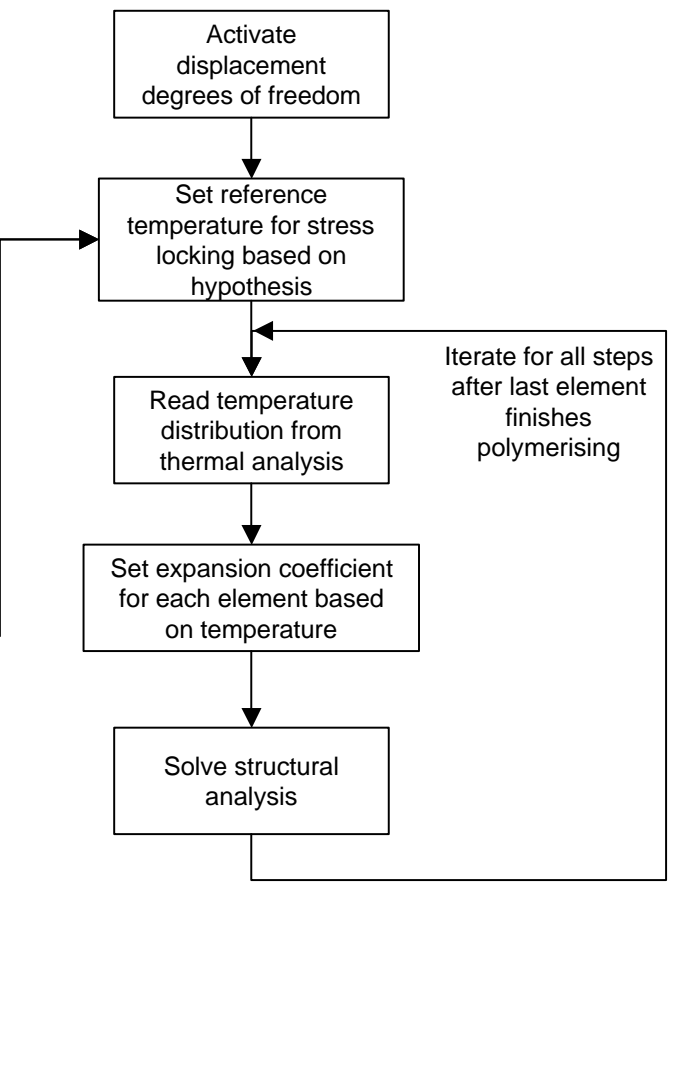

Fig. 2. Algorithm for thermoelastic analysis. The thermal analysis is performed first and results are subsequently used as inputs for the structual analysis stage. Reference temperatures for shrinkage are set according to either peak temperature or temperature upon completion of polymerisation. Iteration in the structural portion is required because of the use of a bilinear expansion coefficient - element temperature must be compared during each step to temperature at which change of coefficient occurs $\left(43^{\circ} \mathrm{C}\right.$ for this study).

mately double the amount (Table 2). Complete cracking of the cement layer was observed in the proximo-lateral region of specimen 1 and in the mid- to disto-lateral and disto-medial regions of specimen 3. Cracks were predominantly oriented normal to the interfaces, although many short cracks, which often originated from pores, were oriented in other directions. Cracks were also observed to originate from both stem-cement and bone-cement interfaces. Initiation sites near the interfaces were difficult to discern due to a high degree of staining at the bone-cement interface, and regions of numerous small pores near portions of the stem-cement interface.

\subsection{Temperature and polymerisation history}

Cement temperature rose rapidly between 6.7 and 10 min, after which it cooled quickly (Fig. 4a) with the rate of cooling dependent on deviation from ambient conditions. The middle of the cement layer exhibited greater and more rapid temperature rise than cement near the interfaces (Fig. 4b). Temperature rise was lowest at the stem-cement interface because heat loss was greatest here, while the bone-cement interface experienced somewhat higher temperature rise due to the bone's lower heat transfer coefficient. The time taken to reach the peak temperature increased slightly for cooler regions; the stem-cement interface reached it last (Fig. 4b), but, in general, the time at which the peak occurred was spread over a relatively small interval Fig. 5 - the entire cement layer reached its peak temperature within a time interval of $38 \mathrm{~s}$. The throughlayer temperature distribution was approximately uniform over the length of the observable cement (Fig. 4c) at the time that the peak occurred, with temperatures along the length of the central region of the layer ranging between approximately $46-53^{\circ} \mathrm{C}$ (Fig. 4c). Temperature of the PMMA at the end of polymerisation ranged between $27-29^{\circ} \mathrm{C}$. Complete polymerisation of the entire acrylic cement occurred almost simultaneously, with $97.5 \%$ of cement elements polymerising before $697 \mathrm{~s}$. 


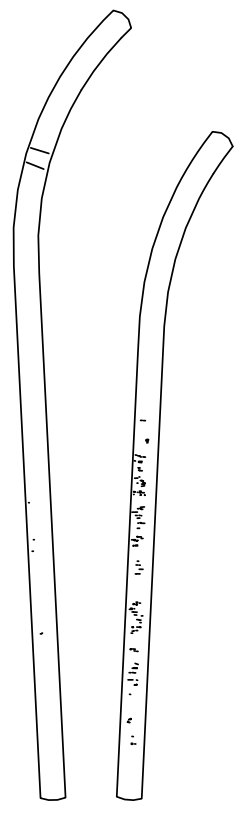

\#1

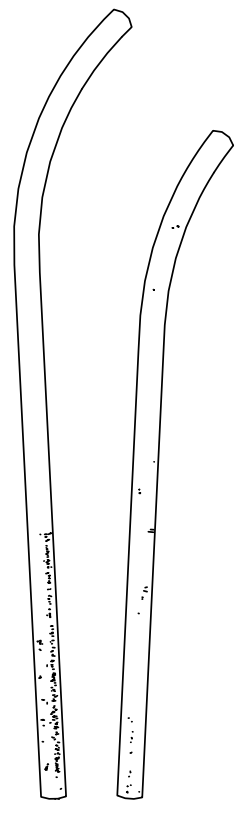

\#2

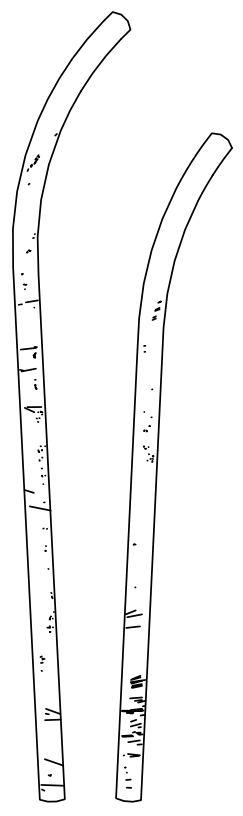

\#3

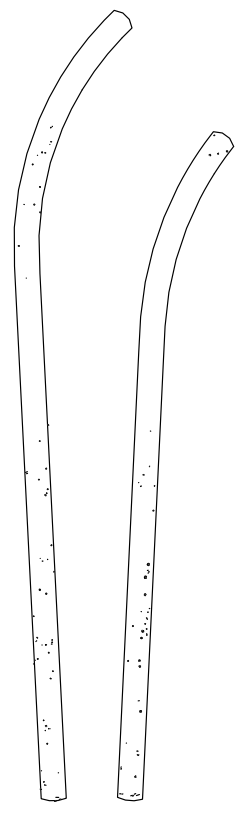

\#4

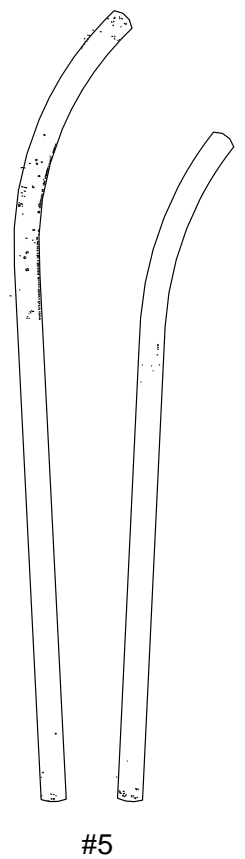

Fig. 3. Pre-load cracks for each of the five specimens. Dots and lines indicate cracks.

Table 2

Number of cracks and sum of crack lengths for each specimen

\begin{tabular}{llllll}
\hline Specimen no. & 1 & 2 & 3 & 4 & 5 \\
\hline No. cracks & 136 & 218 & 173 & 350 & 315 \\
Sum of crack lengths & 38.332 & 31.111 & 84.136 & 19.319 & 34.756 \\
\hline
\end{tabular}

\subsection{Shrinkage stresses}

Whether stress-locking was implemented at the peak temperature or at the end of polymerisation had a pronounced effect on the predicted residual stress in the cement. The maximum principal stresses are in the range of $1-2 \mathrm{MPa}$ for the case of shrinkage from the temperature at the end of polymerisation, whereas they are in a range of 4-7 MPa for the case of shrinkage from the maximum temperature reached (compare Fig. 6 (a) and (b)). The effect of the inclusion of porosity in the stress calculation had a pronounced effect on maximum stress (Fig. 6(c)), resulting in increases of approximately three times the predicted maximum principal stress in many regions.

The maximum residual stresses were predominantly directed parallel to the interfaces, which fits the experimental observation of crack direction, as shown in Fig. 7. Some deviation occurs for short cracks radiating from pores.

Shrinkage also creates normal tensile stresses on the interfaces. These were predicted to range from 1.6 to $3.6 \mathrm{MPa}$ on the lateral stem-cement interface (Fig. 8). Shear stresses in the longitudinal direction at the same interface were low: approximately $-0.2-0.4 \mathrm{MPa}$ (Fig. 8); those in the direction perpendicular to the exposed cement surface were somewhat higher, at approximately $-0.8 \mathrm{MPa}$ (Fig. 8). The same pattern of stress was found for the medial stem-cement interface.

\section{Discussion}

That the stress and crack orientations are mutually orthogonal (Fig. 7) give a strong indication that residual stresses initiate cracks prior to mechanical loading. The level of pre-cracking supports the conclusion that stresses are relatively high, and suggests that the peak temperature is probably the correct reference point for residual stress calculations. Some experimental data exists to support the case of shrinkage from peak temperature - Whelan et al. (2000), using embedded fibre optic sensors, attempted to measure strain within the interior of a curing block of PMMA and did not find strain in the sensor until the peak temperature was reached.

Limitations apply to both physical and computational models. As the cement mantle is modelled by layers, the 

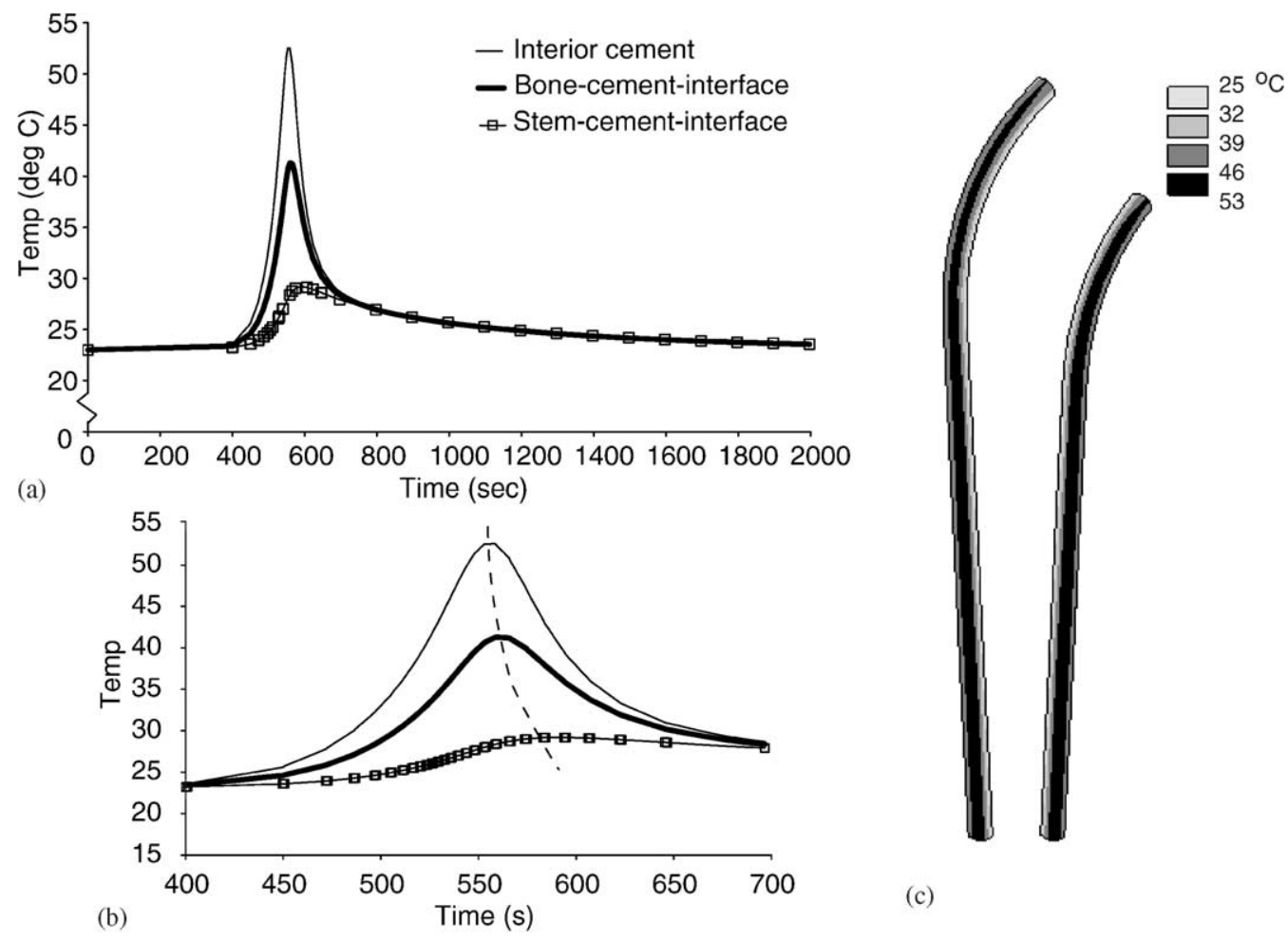

Fig. 4. (a) Temperature history at three points through the cement thickness, between stem-cement and cement-bone interfaces; (b) time-magnified view of temperature pulse to illustrate peak temperature of each region occurring at different times; (c) contour plot of temperature in viewable cement for iteration when highest temperature was reached $(t=536 \mathrm{~s})$.

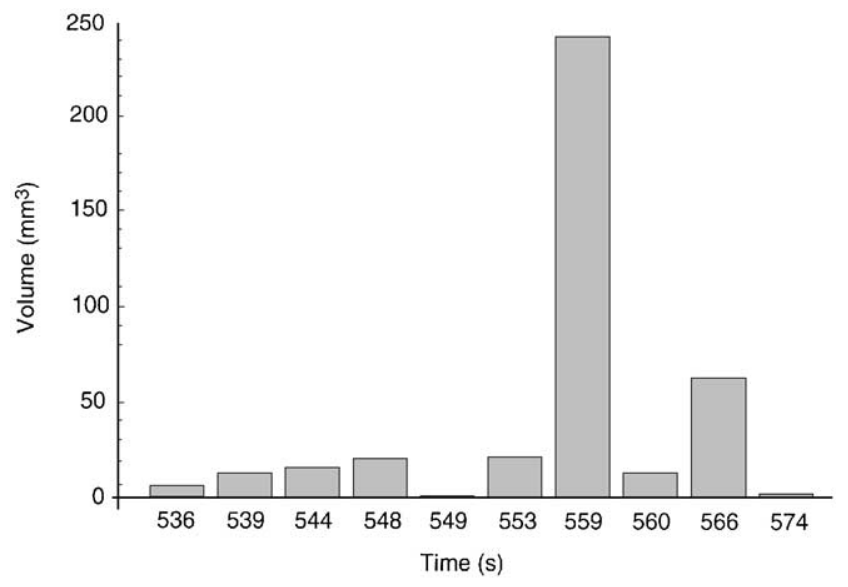

Fig. 5. Histogram showing volumes of cement that reached their peak temperature at different times. Time intervals are not uniform because time-step procedure was based on fastest polymerising element. Notice that most cement peaks at $559 \mathrm{~s}$ while the entire interval is $38 \mathrm{~s}$.

effect of hoop stress is not incorporated in either model. Because the dye penetrant needs access to surfaces in order to stain cracks, only cracks that intersect with the exposed surface can be measured. Another understimation of cracks relates to the use of an orthographic projection of the crack to measure its length; i.e. there is no guarantee that the longest projection of the crack is being imaged. The measurement method is therefore conservative in its estimate of summed length of cracks. Also, to retain transparency, cement without radioopaque filler or other additives was used. In the computational model there is significant uncertainty with respect to the thermal properties of cancellous bone and its interface with the cement, as well as the stemcement interface - this has been mentioned previously by others (Huiskes, 1980; Starke et al., 1997). Furthermore, the bone in this model was at room temperature (approximately $23^{\circ} \mathrm{C}$ compared with $37.5^{\circ} \mathrm{C}$ for the physiological case). Therefore, Less shrinkage might be expected for the in vivo case. However, the higher ambient in vivo temperatures would likely result in an increased temperature pulse - if large enough, this could compensate for the higher final temperature and result in a similar temperature drop as for our in vitro case. A further limitation could be the arbitrarilyassigned initial polymerisation rate; however, since the heat generation rate depends on the total polymerisation fraction, which is set as a $5 \%$ increment, rather than its rate (Eq. (2)), subsequent polymerisation is insensitive to this assumption. Finally, the model calculates stress due to thermal shrinkage from element reference temperatures but these temperatures occur at different times for different elements. An element which has just begun to lock-in stress, but is surrounded by others 


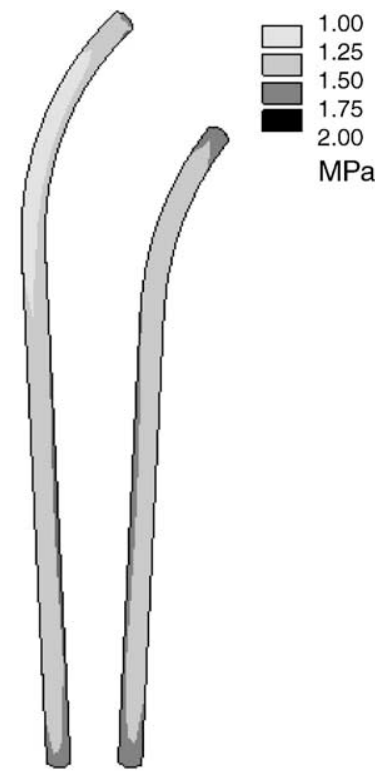

(a) Shrinkage from end of polymerisation

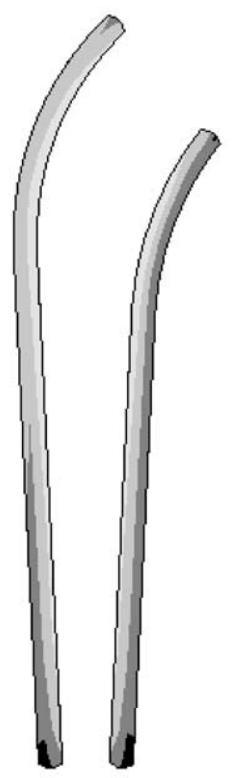

(b) Shrinkage from max polymerisation temperature
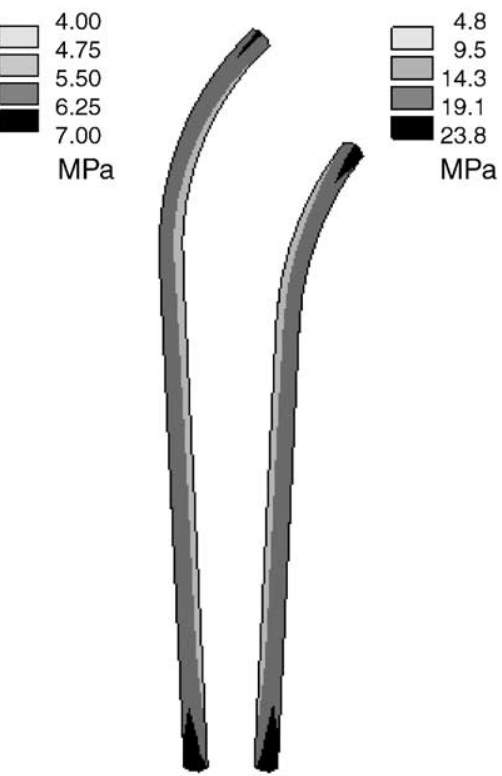

(c) Shrinkage from max polymerisation temperature -pores included

Fig. 6. Maximum principal stress in cement cut-outs for shrinkage of each element relative to (a) its temperature at the time it completed polymerisation, (b) its maximum temperature achieved during polymerisation, and (c) the maximum stress for a uniform pore distribution for stress state of case (b).

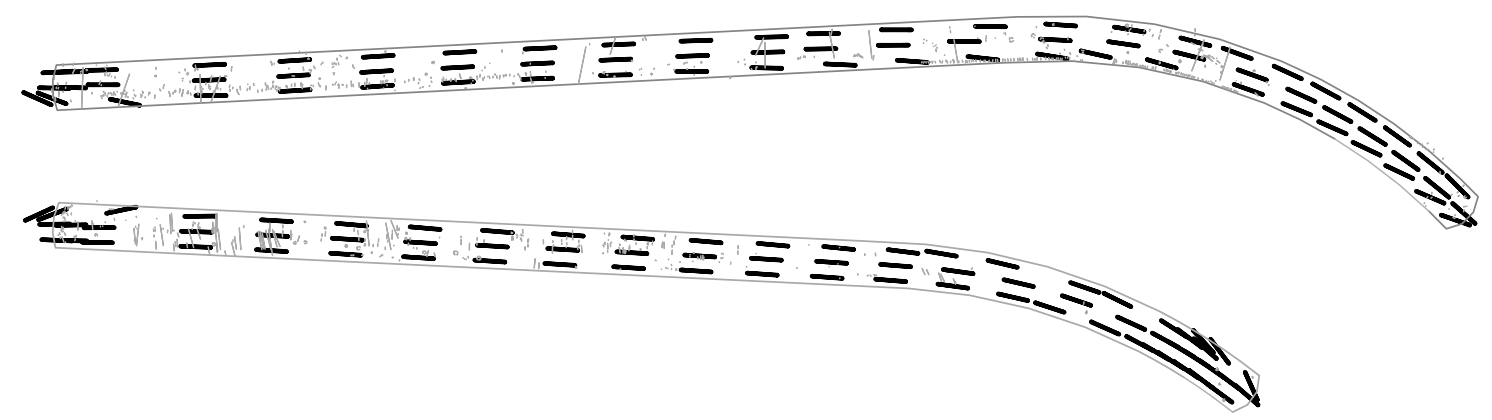

Fig. 7. Plot of maximum principal stress vectors (heavy lines) superimposed on combined crack distribution (light lines) for all five specimens.

which have not yet stress-locked, may not experience significant resistance to shrinkage and little stress would be induced in it. The model is unable to account for this and assumes that elements which have not yet stresslocked will provide the same resistance to neighbouring elements as those that have. However, as the time span for stress-locking is quite small (Fig. 5), the effect will not be too severe. To overcome this deficiency it would be necessary to account for the changing material properties during polymerisation. Such a constitutive relation for chemically hardening materials has been proposed by Shaffer and Levitsky (1974) and implemented for bone cement by Ahmed et al. (1982a).
The thermal analysis predicted differing rates of temperature rise, as well as different peak temperature times, within the mantle, which agrees with the results of other studies, e.g. Meyer et al. (1973), Baliga et al. (1992), and Starke et al. (1997). Other studies have predicted a maximum rise of $45-50^{\circ} \mathrm{C}$ (Huiskes, 1980), $40^{\circ} \mathrm{C}$ (Baliga et al., 1992), and $36-45^{\circ} \mathrm{C}$ (Starke et al., 1997). Peak temperature rise for the observable cement of our physical model was approximately $30^{\circ} \mathrm{C}$, which is therefore between $6^{\circ} \mathrm{C}$ and $20^{\circ} \mathrm{C}$ lower than the predicted in vivo temperature rise from the aforementioned studies. Thus, the predicted temperature drop of our model, in spite of its lower final temperature, is 


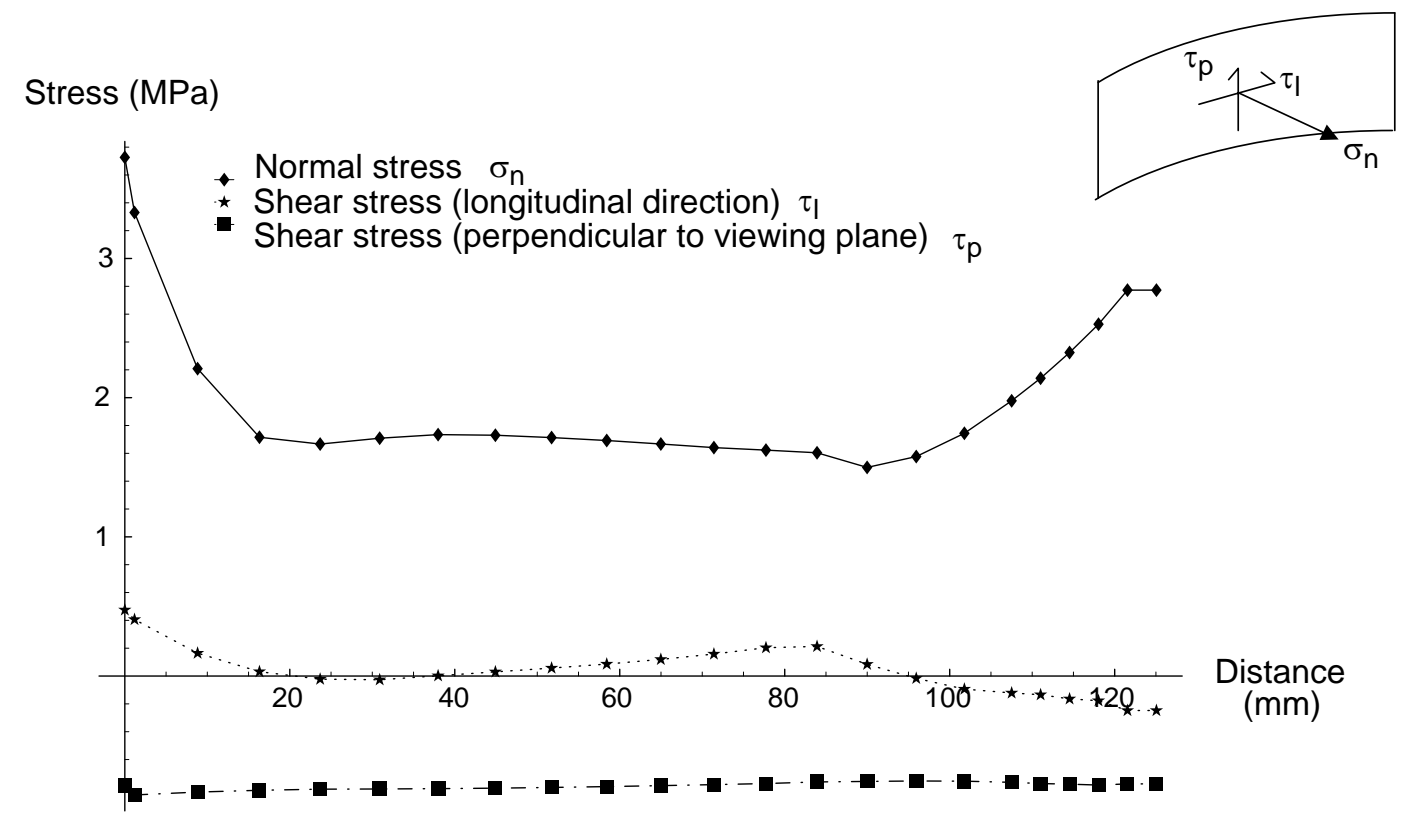

Fig. 8. Plot of normal and shear interface stresses (from distal to proximal) along lateral stem-cement interface for case of shrinkage from maximum temperature. Inset illustrates local interface coordinate system and stress legend.

likely to be of similar proportions to that occurring in vivo. If the drop from peak temperature is a controlling factor in residual stress generation, then the observable cement of the the physical model could be expected to develop similar residual stress, and hence pre-load cracking, as occurs in vivo (a higher temperature rise $\left(48^{\circ} \mathrm{C}\right)$ was predicted below the distal tip of the stem but this region was not observable for the purposes of crack measurement).

If shrinkage is the primary mechanism of stress generation, then a number of factors should further influence the level of stress generated:

(i) The expansion coefficent of PMMA is quite high, and is likely to be even higher prior to complete polymerisation - this would have the effect of further increasing residual stresses, especially if stress-locking occurs at the maximum temperature.

(ii) Geometry and boundary conditions of the structure will affect the shrinkage direction. Consider, first, an annulus of cement - if unrestrained, it would shrink radially inward. However, if an implant is placed inside, the cement will constrict around the implant and the interior surface of the cement will be radially compressed against it and, in the absence of the bone, the external surface would be free of stress in the radial direction. However, if a stiff cortical shell could bond to the external cement, it would restrain the cement from shrinking inwards resulting in radial tension. Thus, in a real hip prosthesis cement mantle, radial cement stress can be expected to vary from compression at the stem-cement interface to tension at the cementbone interface, provided a bond can be maintained with the bone. These geometric and interfacial constraints would also induce tensile hoop stresses as the cement shrinks.

A different mode of shrinkage occurs in the physical model presented above. Shrinkage in the transverse and longitudinal directions depend on the total temperature drop in each direction. Consider three points, oriented in a transverse direction, from the central region of the slice (Fig. 9) - because of the temperature gradient in this direction, a lower average temperature change occurs in the transverse direction compared with the perpendicular set of points in the longitudinal orientation. The points in the longitudinal direction are at a more uniform, and higher, average temperature. Greater shrinkage would then occur in the longitudinal direction. Therefore, provided this shrinkage was restrained (e.g. under conditions of plane strain and interfacial bonding), greatest tensile stresses would be expected in the longitudinal direction, as predicted (see Fig. 7).

We predict somewhat higher maximum tensile stresses, but still of the same order of magnitude, as those predicted by other authors; i.e a range of 4-7 MPa compared with a range of 1-5.5 MPa found in: Huiskes (1980), Mann et al. (1991), and Ahmed et al. (1982a). The level of shrinkage stress for both cases is still an order of magnitude below the static strength of the cement (approx. $25 \mathrm{MPa}$ for Simplex-P according to a review by Saha and Pal, 1984), suggesting that pre- 


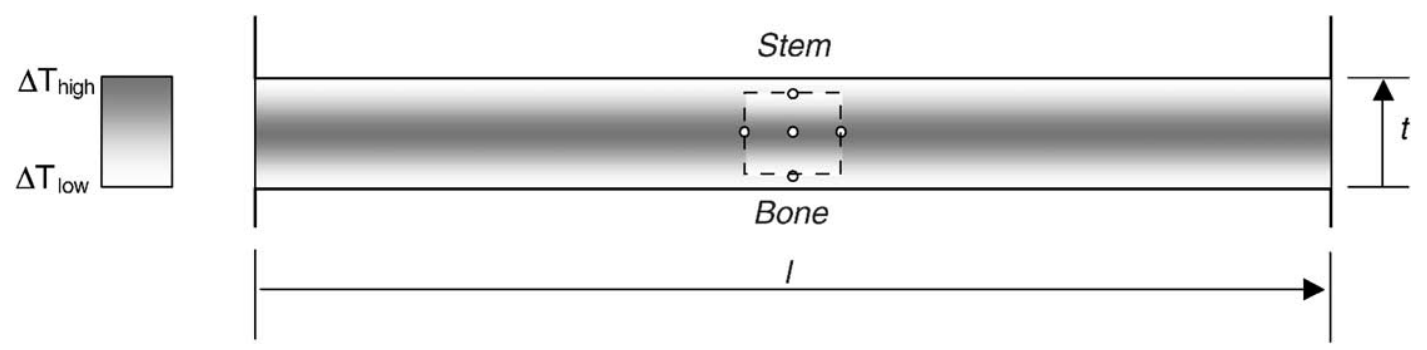

Fig. 9. Schematic illustrating difference between transverse and longitudinal temperature change in a longitudinal slice of cement - the average temperature change of three points in a representative square at the centre of the bar depends on which orientation is examined. Effective temperature drop is thus greater for the longitudinal direction (legend on left of figure illustrates peak temperature change - i.e. peak temperature occurs in central region).

cracking is dependent on a combination of factors, rather than residual stress alone. One such factor is likely to be porosity - this was demonstrated by the estimation of stress around a pore for each element (Fig. 6 (c)) which raised the stress very close to the ultimate tensile strength of the material. This method of stress calculation did not include interaction effects so, in practice, stresses could be even higher around clusters of pores. As porosity takes on an apparently random distribution, at least away from the cement-metal interface, it would be expected that pre-load crack distributions would also appear to be random and would have higher densities in regions with pores. Such a random distribution of cracks is evident from the data for the individual specimens (see Fig. 3) but any study of direct correlation with pores could not be undertaken as the pore distributions were not quantified.

Another possible initiation site for cracks is the bonecement interface - the interdigitated cement is likely to contain stress concentrations which may, possibly in combination with nearby pores, be sufficient to initiate cracks in the shrinking cement. Unfortunately, it was not possible to observe any such behaviour directly, as this interface stained so heavily that it became opaque (due to easier access to the dye penetrant through the cancellous bone).

In contrast to the nonporous stress predictions for the bulk cement, magnitudes of normal interface stresses (1.6-3.6 MPa) were of the same order of magnitude as, although lower than, static interface strength; e.g. 6.9 $\mathrm{MPa}$ for ultimate tensile strength (Raab et al., 1981), and could be expected to accelerate interfacial debonding upon cyclic loading of the specimen. This interface was difficult to observe in the specimens due to increased opacity, created mainly by shadows from the high density of pores in this region, and so no definitive assessment of interface integrity could be made. As the stem had a matt surface finish ( $\mathrm{Ra} \leqslant 3 \mu \mathrm{m})$, and hence relatively high strength, the interface was expected to remain bonded under the predicted interface stresses. If debonding were to occur it would be expected to relieve residual stresses since the cement would not be restrained from shrinking. For the in vivo case this is not likely to be an issue since, as mentioned above, an annular cement mantle shrinking around an implant is likely to result in radial compressive stresses at the implant interface, as predicted by Huiskes (1980).

The presence of shrinkage stress immediately postoperatively can create damage only, it seems, if pores are present. The importance of vacuum mixing would seem to be emphasised by the present results, since it decreases the number of pores and increases the average fatigue life (Murphy and Prendergast, 2000). Similarly, methods to reduce interfacial porosity, such as preheating the stem (Bishop et al., 1996) could be explored using the present analysis since the amount of conduction towards the stem would be reduced by increasing stem temperature; however, the beneficial effect of reduced interface porosity may be offset by the likely increase in magnitude of the temperature pulse. The use of lower exotherm cements, which would result in a reduced temperature pulse, could also be a method of reducing shrinkage stresses but experience with cements such as Boneloc (Biomet, UK) indicate that it may be difficult to develop such a cement with sufficient fatigue strength (Nilsson and Dalen, 1998; Walczak et al., 2000).

In conclusion, residual stress is a factor in pre-load cracking of cement mantles of orthopaedic joint replacements but requires additional factors, such as porosity, stress concentrations, or excessive heat generation, to initiate large cracks. The peak temperature reached for a given region of cement appears to have a significant effect on the level of residual stress that occurs, indicating that control of polymer/monomer ratios as well as ambient conditions during polymerisation are critical in controlling the phenomenon of preload cracking due to shrinkage.

\section{Acknowledgements}

The authors would like to acknowledge the Standards Measurements and Testing Program of the European 
Comission (Contract No. SMT4-CT96-2076) for partly funding the work, and the MediLink project of the PRTLI programme for financial support of one of us (A.B. Lennon). The Department of Mechanical Engineering, University College Dublin, who kindly allowed us to use their optical comparator and prep room.

\section{References}

Ahmed, A.M., Pak, W., Burke, D.L., Miller, J., 1982a. Transient and residual stresses and displacements in self-curing bone cement Part II: thermoelastic analysis of the stem fixation system. Journal of Biomechanical Engineering 104, 28-37.

Ahmed, A.M., Pak, W., Burke, D.L., Miller, J., 1982b. Transient and residual stresses and displacements in self-curing bone cement Part I: characterization of relevant volumetric behaviour of bone cement. Journal of Biomechanical Engineering 104, 21-27.

Baliga, B.R., Rose, P.L., Ahmed, A.M., 1992. Thermal modelling of polymerizing polymethylmethacrylate, considering temperaturedependent heat generation. Journal of Biomechanical Engineering $114,251-259$.

Bishop, N.E., Ferguson, S., Tepic, S., 1996. Porosity reduction in bone cement at the cement-stem interface. Journal of Bone and Joint Surgery $78 \mathrm{~B}(3), 349-356$.

Harrigan, T.P., Harris, W.H., 1991. A three-dimensional non-linear finite element study of the effect of cement-prosthesis debonding in cemented femoral total hip components. Journal of Biomechanics 24 (11), 1047-1058.

Huiskes, R., 1980. Some fundamental aspects of human joint replacement. Acta Orthopaedica Scandinavica 185, 10-208.

Huiskes, R., 1993. Mechanical failure in total hip arthroplasty with cement. Current Orthopaedics 7, 239-247.

Huiskes, R., Verdonschot, N., 1997. Biomechanics of Artificial Joints: The Hip. In: Mow, V.C., Hages, W.C. (Eds.), Basic Orthopaedic Biomechanics, 2nd ed. Lippincott-Raven Publishers, Philadelphia, pp. 395-460.

Kine, B.B., Novak, R.W., 1987. Acrylic and Methacrylic Ester Polymers. In: Mark, H.F., Bikales, N.M., Overberger, C.G., Menges, G., Kroschwitz, J.I. (Eds.), 2nd ed., Encyclopedia of Polymer Science and Engineering, Vol. 1. John Wiley \& Sons, New York, pp. 234-299.

Lennon, A.B., McCormack, B.A.O., Prendergast, P.J., 1998. Development of a physical model of a cemented hip replacement for investigation of cement damage accumulation. In: 11th Conference of the European Society of Biomechanics, Journal of Biomechanics 31 (Suppl. 1), 129.

Lennon, A.B., Prendergast, P.J., 2001. Sub-task 6.3: Cement/metal interface fatigue damage accumulation. Final Report for contract
SMT4-CT96-2076 Preclinical Testing of Cemented Hip Replacement Implants: Pre-normative Research for a European Standard. Nijmegen, The Netherlands.

Mann, K.A., Bartel, D.L., Wright, T.M., Ingraffea, A.R., 1991. Mechanical characteristics of the stem-cement interface. Journal of Orthopaedic Research 9 (6), 798-808.

McCormack, B.A.O., Prendergast, P.J., 1999. Microdamage accumulation in the cement layer of hip replacements under flexural loading. Journal of Biomechanics 32, 467-475.

McCormack, B.A.O., Walsh, C.D., Wilson, S.P., Prendergast, P.J., 1998. A statistical analysis of microcrack accumulation in PMMA under fatigue loading: applications to orthopaedic implant fixation. International Journal of Fatigue 20, 581-593.

Meyer, J.R., Lautenschlager, E.P., Moore, E.K., 1973. On the setting properties of acrylic bone cement. Journal of Bone and Joint Surgery 55-A, 149-156.

Murphy, B.P., Prendergast, P.J., 2000. On the magnitude and variability of fatigue strength in acrylic bone cement. International Journal of Fatigue 22, 855-864.

Nilsson, K.G., Dalen, T., 1998. Inferior performance of Boneloc (R) bone cement in total knee arthroplasty - a prospective randomized study comparing Boneloc (R) with Palacos (R) using radiostereometry (RSA) in 19 patients. Acta Orthopaedica Scandinavica 69 (5), 479-483.

Raab, S., Ahmed, A.M., Provan, J.W., 1981. The quasi-static and fatigue performance of the implant/bone-cement interface. Journal of Biomedical Materials Research 15, 159-182.

Saha, S., Pal, S., 1984. Mechanical properties of bone cement: a review. Journal of Biomedical Materials Research 18, 435-462.

Shaffer, B.W., Levitsky, M., 1974. Thermoelastic constitutive equations for chemically hardening materials. Journal of Applied Mechanics 96, 652-657.

Starke, G.R., Birnie, C., van den Blink, P.A., 1997. Numerical modelling of cement polymerisation and thermal bone necrosis. In: Middleton, J., Jones, M.N., Pande, G.N. (Eds.), Third International Symposium on Computer Methods in Biomechanics and Biomedical Engineering. Gordon and Breach, London, pp. 163172.

Tepic, S., Soltesz, U., 1998. Fatigue of bone cement with simulated stem interface porosity. Journal of Materials Science - Materials in Medicine 9 (12), 707-709.

Walczak, J.P., D’Arcy, J.C., Ross, K.R., James, S.E., Bonnici, A.V., Koka, S.R., Morris, R.W., 2000. Low-friction arthroplasty with Boneloc bone-cement - outcome at 2 to $4 \mathrm{yr}$. Journal of Arthroplasty 15 (2), 205-209.

Whelan, M.P., Kenny, R.P., Cavalli, C., Lennon, A.B., Prendergast, P.J., 2000. Application of optical fibre Bragg grating sensors to the study of pmma curing. In: Prendergast, P.J., Carr, A.J., Lee, T.C. (Eds.), Proceedings of the 12th Conference of the European Society of Biomechanics. Royal Academy of Medicine in Ireland, Dublin, p. 252 . 